\title{
Valores organizacionais e prazer-sofrimento no trabalho
}

\author{
Ana Magnólia Mendes ${ }^{1}$ \\ Álvaro Tamayo
}

\begin{abstract}
Resumo
$\mathrm{O}$ artigo discute as relações entre valores organizacionais e as vivências de prazer-sofrimento no trabalho. Os valores estão definidos como princípios que guiam a vida da organização e estão estruturados em três dimensões bipolares: autonomiaconservação, estrutura igualitária-hierarquia e harmonia-domínio. O prazer-sofrimento é estudado como um constructo dialético, sendo definido como vivências de sentimentos de valorização, reconhecimento e desgaste no trabalho. A pesquisa foi realizada numa empresa pública de abastecimento e saneamento com 554 empregados. Foram aplicadas escalas para medir os valores organizacionais e o prazer-sofrimento, tendo sido os dados analisados por meio de estatística descritiva e correlações bivariadas. Os resultados indicaram uma preponderância das vivências de prazer, sendo o sofrimento vivenciado moderadamente. O prazer está correlacionado com quatro pólos dos valores organizacionais: autonomia, estrutura igualitária, harmonia e domínio. O sofrimento apresenta correlação negativa com o pólo da autonomia, estrutura igualitária e domínio. Palavras-chaves: Valores organizacionais; prazer e sofrimento no trabalho.
\end{abstract}

\section{Organizational values and pleasure-suffering at work}

\begin{abstract}
The paper discusses the relations between organizational values and pleasure-suffering experience at work. Values are defined as principles that guide organization life and they are structured in three bipolar dimensions: autonomy-conservation, equalitarian structures-hierarchy and harmony-mastery. Pleasure-suffering is studied as a dialectic construct and it is defined as an experience of valorization, recognition and wear out feelings at work. The research was undertaken with 554 employees at a Sanitation Public Company. Scales to measure organizational values and pleasure-suffering were used. Data are analyzed through descriptive statistics and correlation. The results indicated high pleasure and moderate suffering experiences. Pleasure is correlated with four organizational values poles: autonomy, equalitarian structures, harmony and mastery. Suffering presents negative correlation with autonomy, equalitarian structures and mastery poles.

Keywords: Organizational values; pleasure-suffering, work.
\end{abstract}

\section{Introdução}

Os valores no contexto organizacional têm sido estudados tanto nas suas relações com o trabalho propriamente dito, quanto com a cultura organizacional.

Em relação ao trabalho, pesquisas realizadas por Ravlin \& Meglino (1989), Meglino, Ravlin e Adkins (1989), Shoskley e Morley (1989) e Judge \& Bretz (1992) estudam os valores sociais dos indivíduos como realização, tolerância e honestidade enquanto influenciadores das relações no trabalho, indicando a importância da compatibilidade entre valores individuais e valores do trabalho para a auto-estima e satisfação no trabalho. Schwartz (1999) e Ros et all (1999) também identificam nos seus estudos relações conceituais entre os valores individuais e os valores atribuídos ao trabalho.

Em relação à cultura, autores como Deal \& Kennedy (1982), Pascale (1984), Shrivastava (1985), Wiener (1988), Zilbovicis (1988), Schein (1990), Hofstede (1990), Freitas (1991) e Trice \& Beyer (1993) estudam os valores como associados aos objetivos organizacionais, aos processos de socialização constante e integrados a um sistema de normas e regras

${ }^{1}$ Endereço para correspondência:

SQN 206 Bloco K apt. 105, Asa Norte, Brasília- DF, 70862-010.

E-mail: anamag@yawl.com.br compartilhadas. Desenvolvem modelos para identificar a cultura organizacional, mas não especificamente um modelo de estudo dos valores organizacionais.

A partir dessas contribuições teóricas, assume-se como pressuposto que, os valores constituem um dos importantes instrumentos para entender a cultura organizacional. A cultura de uma organização sustenta o processo de socializações por meio do sistema de valores, sendo tais valores vivenciados como uma experiência subjetiva compartilhada, que cria nas organizações a possibilidade de simbolização e mediação das necessidades individuais e organizacionais.

Assim sendo, os valores têm um papel tanto de atender aos objetivos organizacionais, quanto de atender às necessidades dos indivíduos. Os valores organizacionais podem passar mensagens e comportamentos convenientes, levando à naturalização do conteúdo e ao repasse espontâneo para os demais membros, fazendo com que a adesão e a reprodução permitam a liberdade dos indivíduos em aceitar ou não determinados conteúdos ou a eficiência do controle dessa liberdade.

Para fundamentar esses pressupostos, adota-se o modelo dos valores organizacionais, proposto por Tamayo (1996), com base nos estudos dos valores culturais realizados por Schwartz \& Ros (1995) e Schwartz (1999). Os primeiros estudos empíricos da 
percepção dos valores organizacionais foram realizados no Brasil por Gondin (1996) e por Tamayo-Robayo (1997).

A utilização desse modelo significa considerar que os valores não estão definidos a priori e podem ser classificados numa hierarquia, não são fixos, variam de uma organização para outra e até dentro da própria organização. Os valores organizacionais servem à própria sobrevivência da organização e, por essa razão, buscam mediar conflitos para resolver problemas.

Segundo Tamayo (1996), os valores têm por objetivo resolver três problemas: a conciliação de interesses individuais e do grupo; a necessidade de uma estrutura que contemple a definição de papéis, normas e regras para relações e organização do trabalho; e a conciliação entre interesses da organização e do meio social e natural, que se caracteriza pela necessidade de produtividade e sobrevivência da organização que retira do meio a matéria-prima e realiza as trocas comerciais.

Com base na necessidade dessas mediações, o autor propõe três dimensões bipolares para o estudo dos valores organizacionais. Essas dimensões permitem o entendimento da cultura organizacional na medida em que resulta do estudo das dimensões culturais que fazem parte das sociedades.

Dimensão da autonomia e da conservação - A organização que privilegia a autonomia intelectual e afetiva é uma organização que enfatiza, respectivamente, a promoção e a proteção da independência de idéias e o direito do indivíduo procurar sua direção e promoção, além da independência do indivíduo de buscar experiência afetiva positiva. A organização que privilegia a conservação, enfatiza a manutenção do status quo e as restrições das ações que podem causar ruptura na solidariedade do grupo ou das tradições.

Dimensão da hierarquia e da estrutura igualitária - A organização que prioriza a hierarquia enfatiza a legitimidade da ordem interna e a subordinação dos poderes em relação à alocação de papéis e recursos. Caso a organização priorize o igualitarismo está enfatizando a transcendência dos interesses individuais e organizacionais, em favor de um compromisso consciente, voluntário e responsável para promover o bem-estar de todos.

Dimensão da harmonia e do domínio - A organização que privilegia a harmonia enfatiza o ajustamento constante e harmonioso com o ambiente externo. Privilegiado o domínio, a ênfase é buscar prosperidade por meio da auto-afirmação ativa para enfrentar as mudanças externas e para dominar o ambiente social e natural.

A classificação de uma organização nessas dimensões revela as prioridades axiológicas da organização, a sua base motivacional predominante, e a percepção dos trabalhadores dessas prioridades, fato que define a sua relação com a organização e o seu comportamento cotidiano em função da representação cognitiva que ele tem da organização.

Neste artigo, o estudo dos valores organizacionais não será realizado de forma isolada, mas integrado à variável prazer-sofrimento. $\mathrm{O}$ prazer-sofrimento vem sendo estudado por Dejours (1993,1994,1998), Mendes e Abrahão (1996), Mendes (1995, 1996, 1999) e Mendes e col.(2000), articulados à variável organização do trabalho, caracterizada pelo conteúdo da tarefa e relações socioprofissionais, a qual exerce um impacto no funcionamento psíquico do trabalhador gerando prazersofrimento dependendo do quanto a tarefa é significativa para o trabalhador e se as relações com colegas e chefias são ou não de reconhecimento, cooperação, confiança e solidariedade. $O$ prazer-sofrimento inscreve-se numa relação subjetiva do trabalhador com seu trabalho, que implica intersubjetividade no momento em que esse sujeito passa a relacionar-se com outros, sendo os valores, como princípios que guiam a vida da organização, um dos elementos responsáveis pela socialização das normas e regras, que definem formas específicas do trabalhador vivenciar sua tarefa e compartilhar suas relações sociais, afetivas e profissionais no contexto organizacional.

Os valores fazem parte de uma dialética de manutenção e de transformação dos comportamentos humanos pela socialização e aprendizagem permanentes, sendo, por isso, valiosos para as instituições que desejam modelar comportamentos em função de seus interesses, que podem ser favoráveis ou não às vivências de prazer e de sofrimento. Os valores das organizações podem ser uma das fontes geradoras de prazer no trabalho, desde que favoreçam uma organização do trabalho flexível, marcada pela possibilidade de negociações das regras e normas dos processos de trabalho, com participação dos trabalhadores e gestão coletiva das necessidades individuais e organizacionais.

Quando os valores influenciam uma organização do trabalho marcada pela ausência de críticas e negociações das normas e regras, levando à naturalização das situações e à criação de verdades inquestionáveis, pode ter lugar o sofrimento no trabalho. O bloqueio da convivência com as singularidades pode levar à alimentação e/ou produção de um sofrimento, muitas vezes, mascarado por defesas, o que dificulta a identificação das suas causas e as possibilidades para sua transformação, imperando desta forma, no espaço de trabalho, o sofrimento e não o prazer.

As vivências de prazer-sofrimento formam um único constructo composto por três fatores: valorização e

Psico-USF, v.6, n.1, p.39-46, jan./jun. 2001 
reconhecimento, que definem o prazer; e desgaste com o trabalho, que define o sofrimento. O prazer é vivenciado quando são experimentados sentimentos de valorização e reconhecimento no trabalho. A valorização é o sentimento de que o trabalho tem sentido e valor por si mesmo, é importante e significativo para a organização e a sociedade. O reconhecimento é o sentimento de ser aceito e admirado no trabalho e ter liberdade para expressar sua individualidade. O sofrimento é vivenciado quando experimentado o desgaste em relação ao trabalho, que significa a sensação de cansaço, desânimo e descontentamento com o trabalho. Assim sendo, prazersofrimento são vivências de sentimentos de valorização, reconhecimento e/ou desgaste no trabalho.

O modelo tridimensional dos valores organizacionais pressupõe um funcionamento organizacional específico, que pode ser favorável ou não à vivência de prazersofrimento e ao espaço oferecido aos trabalhadores para manejar as situações que fazem sofrer. Essas características para o funcionamento organizacional, originadas nas dimensões dos valores organizacionais, podem estar presentes de forma isolada, integrada, e até contraditória, inclusive dentro de uma mesma organização baseada na idéia de subculturas. Também, considera-se que o funcionamento organizacional pode trazer combinações dos pólos dos valores organizacionais diferentes, dependendo da situação vivenciada pela organização num dado momento.

Ressalta-se ainda, que o trabalhador pode perceber os pólos dos valores organizacionais de uma forma que não contemple totalmente a realidade organizacional, tendo em vista os processos simbólicos da relação indivíduo-cultura, sendo a integração entre a percepção dos valores organizacionais, a realidade e a subjetividade própria a cada trabalhador, as condições favoráveis ao prazer ou ao sofrimento no trabalho.

Isto posto, a pesquisa busca identificar quais as relações entre a percepção dos pólos dos valores organizacionais e o prazer-sofrimento no trabalho.

\section{Método}

\section{Amostra}

A pesquisa foi realizada numa empresa pública de abastecimento e saneamento do DF. Participaram da pesquisa 554 empregados. A amostra foi estruturada e aleatória, tendo participado da pesquisas empregados das áreas administrativa, técnica, operacional e comercial. A amostra está distribuída de acordo com as características apresentadas na tabela 1.

Tabela 1. Percentual da distribuição da amostra segundo escolaridade, sexo, tempo de serviço.

\begin{tabular}{|c|c|c|c|}
\hline Escolaridade & $(\%)$ & Sexo & Tempo de serviço (\%) \\
\hline 2o grau incompleto & 7,1 & Masculino 65,3 & até 5 anos $\quad 52,6$ \\
\hline $2^{\circ}$ grau completo & 41,0 & Feminino 28,7 & 6 a 10 anos \\
\hline Superior incompleto & 16,0 & & 11 a 15 anos \\
\hline Superior & 31,4 & & Acima de 15 anos 6,1 \\
\hline Total & 95,5 & 94,0 & 85,6 \\
\hline
\end{tabular}

\section{Instrumentos}

Os valores organizacionais foram medidos por meio do Inventário de Valores Organizacionais (IVO), com escala de 7 pontos e 66 itens, validado por Tamayo, Mendes e Paz (2000).

Os valores do eixo da autonomia são criatividade, curiosidade, eficácia, eficiência, modernização, qualidade, reconhecimento, competência, prazer, inovação, realização, estimulação e liberdade. $\mathrm{Na}$ conservação, são disciplina, honestidade, lealdade, limpeza, ordem, polidez, prestígio, segurança, sensatez e sigilo. $\mathrm{Na}$ estrutura igualitária, democracia, descentralização, justiça, qualificação dos recursos humanos, sociabilidade, co-gestão, coleguismo, igualdade, cooperação, dinamismo, independência, autonomia, comunicação, respeito e eqüidade. $\mathrm{Na}$ bierarquia, fiscalização, hierarquia, obediência, poder, pontualidade, rigidez, supervisão e tradição. $\mathrm{Na}$ harmonia, integração interorganizacional, interdependência, ética, parceria, tolerância, intercâmbio, preservação, equilíbrio, preservação da natureza, respeito à natureza, espontaneidade, iniciativa. No domínio, ambição, audácia, competitividade, domínio, previsibilidade, produtividade, satisfação dos clientes e sucesso.

O prazer-sofrimento foi medido pela Escala de Prazer-Sofrimento no Trabalho (EPST), de 5 pontos com 37 itens, validada por Mendes (1999). É composta pelos fatores valorização e reconbecimento que medem o prazer e desgaste, que mede o sofrimento.

O Inventário de Valores Organizacionais (IVO) e da Escala de Prazer-Sofrimento no Trabalho (EPST) foram aplicados conjuntamente na amostra.

\section{Análise dos dados}

Os dados obtidos na aplicação dos instrumentos de medida foram submetidos à análise, utilizando o programa de estatística SPSS (Statistical Package for 
Social Science, versão5.0). Inicialmente foram realizadas análises exploratórias com o objetivo de identificar os missing e autliers, tendo sido os missing substituídos por valores médios e os autliers retirados da amostra, que de 554 participantes passou a ser de 551 .

Para o tratamento das variáveis foram calculadas as médias para os fatores do prazer-sofrimento e para cada um dos pólos dos valores organizacionais. Analisou-se a curva da distribuição dos escores médios para cada um dos fatores e pólos a fim de definir o comportamento das variáveis, identificando o predomínio de uns fatores em relação aos outros. Para avaliar a significância da diferença das médias obtidas nos três fatores do sofrimento e nas três dimensões dos valores organizacionais, realizouse análise de variância. A verificação da influência dos pólos dos valores organizacionais no prazer-sofrimento no trabalho foi verificada por meio de análise correlacional.

Para esses procedimentos foram excluídos da amostra os casos que apresentavam resultados extremos com média máxima, ao mesmo tempo, nos pólos opostos de cada uma das dimensões, autonomiaconservação, estrutura igualitária-hierarquia, harmoniadomínio, resultando 426 casos analisados e 125 excluídos. Esta exclusão teve por objetivo retirar casos que contribuem para contaminar a polaridade das dimensões dos valores.

\section{Resultados}

Os resultados obtidos na Escala de PrazerSofrimento são apresentados na tabela 2, e indicam a média total da amostra nos três fatores do instrumento.

Tabela 2. Médias e desvio-padrão da amostra para os fatores valorização, desgaste e reconhecimento ( $\mathrm{n}=537)$

\begin{tabular}{l|c|c}
\hline Fatores & Médias & Desvio padrão \\
\hline Valorização & 3,9 & 0.65 \\
\hline Reconhecimento & 3,4 & 0.69 \\
\hline Desgaste & 2,4 & 0.65 \\
\hline
\end{tabular}

A diferença entre as médias nos três fatores é significativa, conforme resultados da análise de variância, que apresenta níveis de significância de $\mathrm{p}$ $\leq 0,0001(\mathrm{~F}<2,2$ e $\mathrm{Gl}<73)$. A EPST é uma escala de 5 pontos, apresentando um ponto médio em 3 . Os resultados diferenciam-se para os fatores do prazer, estando ambos acima do ponto médio, e para o fator desgaste do sofrimento que se encontra abaixo do ponto médio.

Esses resultados indicam que na organização existe um predomínio da vivência de prazer e uma vivência de sofrimento relativamente moderada pela proximidade do resultado do fator desgaste com o ponto médio, significando que ambas existem para os trabalhadores dessa organização.

Os resultados do IVO têm como objetivo classificar a organização nos pólos dos valores organizacionais, identificando as prioridades axiológicas que guiam a vida da organização. $\mathrm{Na}$ tabela 3, apresentam-se as médias e desvios padrões para cada um dos pólos das três dimensões dos valores organizacionais.

Tabela 3. Médias dos pólos das três dimensões dos valores Organizacionais ( $\mathrm{n}=537$ )

\begin{tabular}{|c|c|c|c|c|c|c|c|c|c|c|c|}
\hline \multicolumn{2}{|c|}{ Autonomia } & \multicolumn{2}{|c|}{ Conservação } & \multicolumn{2}{|c|}{ Hierarquia } & \multicolumn{2}{|c|}{$\begin{array}{l}\text { Estrutura } \\
\text { igualitária }\end{array}$} & \multicolumn{2}{|c|}{ Harmonia } & \multicolumn{2}{|c|}{ Domínio } \\
\hline $\mathrm{X}$ & DP & $\mathrm{X}$ & DP & $\mathrm{X}$ & DP & $\mathrm{X}$ & DP & $\mathrm{X}$ & DP & $\mathrm{X}$ & DP \\
\hline 4,0 & 1.16 & 4,3 & 1.03 & 4,0 & 0.97 & 3,6 & 1.18 & 3,8 & 1.06 & 4,0 & 1.05 \\
\hline
\end{tabular}

Realizando a análise de variância, verificaram-se diferenças significativas de $\mathrm{p} \leq 0,0001(\mathrm{~F}<2,5$ e Gl $<$ 78) para a combinação dos pólos opostos das três dimensões dos valores organizacionais. $\mathrm{Na}$ dimensão autonomia-conservação, de acordo com as médias existe o predomínio da percepção no pólo da conservação. $\mathrm{Na}$ dimensão hierarquia-estrutura igualitária, predomina a hierarquia, e na dimensão harmonia-domínio, predomina o domínio.

Em termos de prioridades axiológicas pode-se dizer que a organização é percebida enfatizando em ordem decrescente a conservação, hierarquia, domínio, autono- 
mia, harmonia e estrutura igualitária. Importante é destacar o predomínio da conservação e as diferenças entre as dimensões, surgindo como prioridades axiológicas da organização, a conservação, hierarquia e domínio.
Os resultados das correlações entre os pólos dos valores organizacionais e o prazer-sofrimento são apresentados na tabela 4 .

Tabela 4. Correlações de Pearson entre os fatores do Prazer-Sofrimento e os pólos dos valores organizacionais $(n=416)$

\begin{tabular}{l|c|c|c}
\hline & Valorização & Reconhecimento & Desgaste \\
\hline Autonomia & $.23^{*}$ & $.30^{*}$ & $-.27^{*}$ \\
\hline Conservação & .18 & $.23^{*}$ & -.13 \\
\hline Estrutura igualitária & $.20^{*}$ & $.27^{*}$ & $-26^{*}$ \\
\hline Hierarquia & .15 & .08 & -.06 \\
\hline Harmonia & $.28^{*}$ & $.29^{*}$ & -.16 \\
\hline Domínio & $.27^{*}$ & $.22^{*}$ & $-19^{*}$ \\
\hline
\end{tabular}$$
*_{\mathrm{p}} \leq .005
$$

De acordo com a tabela, o fator valorização do prazer no trabalho é correlacionado positivamente com os pólos da autonomia, estrutura igualitária, harmonia e domínio. O fator reconhecimento apresenta correlação positiva com os pólos da autonomia, conservação, estutura igualitária, harmonia e domínio. O fator desgaste apresenta correlação negativa com o pólo da autonomia, estrutura igualitária e domínio, prevalecendo para cada uma das dimensões um dos pólos opostos.

\section{Discussão dos resultados}

Os resultados demonstram que o prazer-sofrimento não é excludente, confirmando o aspecto dialético do constructo apresentado na teoria, ainda que para esta organização seja significativo o predomínio do prazer em relação ao sofrimento. Com base nesses resultados pode-se dizer que os trabalhadores dessa organização possivelmente vivenciam prazer porque estabelecem relações significativas com sua tarefa e com os colegas e chefias, aspectos representados no fator valorização e reconhecimento, implicando que essa organização oferece condições necessárias para o trabalho ser fonte de prazer, mesmo que o sofrimento não esteja completamente ausente.

Ao sentir valorização, o trabalhador considera seu trabalho importante para si mesmo, para a empresa e a sociedade, indicando assim, um reforço positivo na auto-imagem, que está relacionada ao orgulho pelo trabalho que faz, à realização profissional, ao sentir-se útil e produtivo. A predominância do sentimento de valorização nos resultados também pode ter relação com o momento da organização, caracterizado por investimentos na produção, na qualidade dos serviços e na sua imagem diante do público. Esses elementos podem ser favoráveis ao sentimento de orgulho e utilidade, que estão na base da valorização.
O reconhecimento pode significar que as relações socioprofissionais são razoáveis. Existem de forma moderada relações de liberdade e de boa convivência com as chefias e os colegas, bem como o espaço para construir um coletivo de trabalho no qual estão presentes as margens de liberdade para ajustar as suas necessidades à tarefa.

Os resultados em relação ao sofrimento indicam uma sensação de desgaste moderada, o que pode ter explicação no tipo de tarefas realizadas. Os trabalhadores que sentem desgaste exercem atividades cansativas, desagradáveis, repetitivas, com mais sobrecarga, o que gera frustrações, desânimo, insatisfação. Também, significa pessoas submetidas a sistemas injustos de avaliação de desempenho, bem como a injustiças ligadas ao exercício do poder.

O predomínio do prazer pode ter fundamento na concepção de que o trabalho, segundo Mendes (1999) é lugar de realização, de identidade, valorização e reconhecimento, sendo a busca do prazer uma constante para todos os trabalhadores na direção de manter o seu equilíbrio psíquico, tendo o sofrimento um lugar que surge a partir das imposições que as condições externas às situações de trabalho impõem aos trabalhadores. Teoricamente, esse predomínio também pode ser explicado pelo pressuposto de que o trabalho é um dos caminhos para o investimento da pulsão por meio da sublimação, que mais aproxima o homem do seu desenvolvimento, segundo Freud (1930/1974). O confronto com uma realidade restritiva é que mobiliza a não-gratificação dessa energia pulsional, gerando conflitos e causando sofrimento. Por isso, é importante a forma como o trabalho é organizado no sentido de oferecer maior margem de liberdade para expressão da sublimação como energia pulsional resignificada que resulta em prazer no trabalho. 
Quanto aos valores organizacionais, os resultados implicam que a organização apresenta um funcionamento baseado nos pólos da conservação, hierarquia e domínio. Isso pode significar um funcionamento organizacional mais voltado para manutenção do statusquo, das tradições, da ordem, disciplina, obediência à autoridade, a satisfação dos clientes, preocupação com o mercado, com a produtividade e com o sucesso organizacional.

Analisando cada um dos pólos em relação aos dilemas mediados pelas dimensões das quais fazem parte, identifica-se que para solucionar o dilema indivíduogrupo, a organização atende mais a necessidades do grupo do que dos indivíduos, privilegiando valores de conservação. $\mathrm{O}$ dilema relativo ao seu funcionamentos interno quanto à definição de normas, papéis e estrutura gerencial atende à necessidade de manter a disciplina e a autoridade, existindo poucos espaços para democracia, participação, descentralização, cooperação e outros valores da estrutura igualitária, tendo em vista o predomínio da hierarquia. Em relação ao dilema organização-ambiente externo, atende mais às necessidades voltadas ao domínio do mercado e qualidade dos produtos, não estabelecendo relações de parceria com outras organizações, como enfatizado nos valores do pólo da harmonia.

A organização, para a maioria dos empregados, comporta-se como mantenedora das tradições, da segurança, da lealdade, da obediência à ordem e autoridade, e da relação sem parceria com o ambiente. Aspectos estes que podem estar em processo de mudança, tendo em vista a percepção da existência dos demais pólos dos valores como a autonomia, a estrutura igualitária e a harmonia.

Assim sendo, o perfil organizacional, valendo-se desses resultados, pode trazer como conseqüências para o trabalho a necessidade de ajustar os trabalhadores aos interesses vigentes da organização, evitando conflitos que gerem alterações no já estabelecido; uma estrutura interna burocrática voltada para divisão de tarefas, separação entre concepção e execução, mecanização e desqualificação, baixo reconhecimento no trabalho e a não valorização dos processos de comunicação, da criatividade e auto-realização.

A percepção desse valores também pode representar a dificuldade dos empregados absorverem as mudanças organizacionais na mesma velocidade que ocorrem de fato, mantendo, por um certo tempo, a imagem já construída da organização. Isso se justifica pelo fato de os valores de conservação, hierarquia e domínio terem sido percebidos na média, significando que há grupos na organização que também a percebem mais voltada para os pólos da autonomia, estrutura igualitária e harmonia, que de alguma forma são valores mais compatíveis com os processos de mudança atuais, implicando a fase de transição que a organização se encontra.

Em relação às correlações entre às dimensões dos valores organizacionais e as vivências de prazer-sofrimento, os resultados para valorização e autonomia permitem concluir que a valorização é vivenciada quando a organização enfatiza a liberdade dos empregados para desenvolver sua autonomia intelectual, buscando criatividade, curiosidade, inovação, realização, estimulação, bem como, encontrando liberdade para execução das tarefas visando à eficácia, eficiência, modernização, qualidade e reconhecimento, além de se valorizarem a competência e o prazer no trabalho. As conseqüências desses valores para o cotidiano de trabalho aproximam-se dos antecedentes da organização do trabalho que gera prazer, pesquisado por Mendes (1996 e 1999), à medida que o trabalho tem sentido e importância tanto para o indivíduo como para a organização, considerando que são valores resultantes ou reforçadores dos sentimentos de utilidade, produtividade quando têm espaço a criatividade, liberdade $\mathrm{e}$ autonomia, o que estabiliza a identidade, fortalecendo a auto-imagem em razão da predominância do sentimento de valorização.

As correlações entre a estrutura igualitária e a valorização significam que o sentimento de valorização também é vivenciado quando a organização é percebida enfatizando valores de democracia, descentralização, justiça, qualificação dos recursos humanos, sociabilidade, co-gestão, coleguismo, igualdade, cooperação, dinamismo, independência, autonomia, comunicação, respeito e eqüidade. Também está correlacionada com o sentimento de valorização a harmonia, que significa a ênfase organizacional na integração interorganizacional, interdependência, ética, parceria, tolerância, intercâmbio, preservação, equilíbrio, preservação da natureza, respeito à natureza, espontaneidade, iniciativa, significando assim, que a valorização depende do posicionamento da organização ante o ambiente, trazendo conseqüências positivas para a auto-imagem do trabalhador, podendo predominar sentimentos de orgulho e admiração pelo que faz, aspectos medidos pelo fator de valorização.

Contraditoriamente, a valorização também correlaciona-se com o domínio, que é pólo oposto à harmonia. Esse fato pode ter explicação nas relações conceituais do significado do domínio para o sentimento de valorização, considerando que são valores que favorecem o sentir-se útil e produtivo à medida que enfatizam a qualidade dos produtos, o sucesso, a satisfação dos clientes, a própria imagem da empresa, o que pode ter como conseqüência uma valorização do empregado pela importância que a 
empresa e a sociedade passam a atribuir ao trabalho realizado no cotidiano. Além do mais, esse foi um dos pólos percebidos pela maioria dos empregados, o que pode significar um momento vivido pela empresa, que, independentemente do tipo de sentimentos gerados no trabalho, será percebido por retratar a realidade organizacional.

O fator reconhecimento aparece correlacionado mais fortemente à autonomia, estrutura igualitária, harmonia, mas também à conservação e ao domínio, pólos opostos à autonomia e à harmonia, respectivamente. Considerando que as correlações mais fortes relacionam-se A autonomia, estrutura igualitária e harmonia, pode-se concluir que o sentimento de reconhecimento acontece quando a organização enfatiza a independência dos empregados, uma estrutura interna que tem flexibilidade na definição dos papéis hierárquicos, nas normas e regras e uma relação com o ambiente de parceria. Esses valores juntos trazem como conseqüência as possibilidades para a estruturação psicoafetiva das relações socioprofissionais, um dos elementos que gera o sentimento de reconhecimento pelos pares e hierarquia, bem como permite o suporte afetivo e social necessário ao fortalecimento da identidade por meio do coletivo de trabalho, do reconhecimento da marca pessoal e da competência no trabalho.

As correlações entre o reconhecimento e a conservação podem ser explicadas nos valores que enfatizam a segurança, estabilidade e coesão grupal, como elementos também favoráveis ao reconhecimento, mesmo que a conservação se oponha à autonomia, certamente, para algumas pessoas, o reconhecimento não está na independência para criar, inovar e realizar-se profissionalmente, mas na conservação do conquistado e estabelecido. $\mathrm{O}$ mesmo pode ocorrer em relação ao domínio, que se opõe à harmonia, mas pode, pela necessidade de competitividade no mercado e supremacia dos produtos e serviços, criar um clima interno favorável para o reconhecimento no trabalho.

Para o desgaste, fator do sofrimento no trabalho, os resultados são significativos em relação à autonomia, estrutura igualitária e domínio. No entanto, são relações negativas, parecendo indicar que, caso a organização não enfatize esses valores, os trabalhadores experimentam desgaste no trabalho, sentindo frustração, insatisfação, desmotivação e falta de entusiasmo com o trabalho, enquanto que, se a organização enfatiza esses valores, os indivíduos experimentam a valorização e o reconhecimento.

\section{Considerações finais}

Como conclusão considera-se que o prazer está correlacionado positivamente com pólos das três dimensões dos valores organizacionais, enquanto o sofrimento correlaciona-se negativamente com esses pólos. Isso pode indicar que a cultura assume um papel de gratificação e realização do desejo, por isso, espaço para o prazer, emergindo o sofrimento quando este prazer não tem mais lugar, não sendo assim, geradora de sofrimento, mas reguladora, à medida que o sofrer mais ou menos, depende do quanto de prazer foi restringido pelas imposições externas.

Os resultados obtidos com a análise desses dados fundamentam a idéia de que o sofrimento dificilmente aparece como predominante no contexto organizacional o que pode ser explicado, pela necessidade inerentes à condição humana do indivíduo buscar constante prazer e evitar o sofrimento, fazendo com que estratégias sejam utilizadas para evitar, minimizar ou transformar o sofrimento. Assim sendo, os valores têm a função de gratificar os desejos mediante o atendimento das necessidades dos indivíduos, tornando-se restritivos quando cristalizados ou rígidos, ao ponto de serem criadas ideologias de comportamento bloqueadoras da liberdade de escolha dos empregados, como pode acontecer com as organizações com estruturas inflexíveis. Por isso, determinados pólos de valores, como a autonomia, estrutura igualitária e harmonia, de acordo com os resultados da pesquisa, são promotores de vivências de prazer, enquanto sua não-existência pode gerar restrição ao prazer e dar lugar ao sofrimento.

Não é possível pelos resultados afirmar que tipo de padrão de funcionamento organizacional seria definidor da vivência de sofrimento. É possível concluir que o prazer e a diminuição ou evitação do sofrimento encontram-se relacionados a um contexto organizacional onde predominam a percepção dos valores do pólo da autonomia, estrutura igualitária e harmonia, que constituem a base para um contexto organizacional mais flexível, resgatando e dando condições para satisfação das necessidades e para o trabalho assumir seu papel de ser fonte de prazer.

Estes resultados, apesar de monstrarem correlações entre valores e prazer-sofrimento, ainda apresentam limitações quanto à explicação de tais correlações, sendo necessário a indicação de mais estudos que venham a aprofundar e clarificar a natureza e intensidade das relações entre as variáveis.

\section{Referências bibliográficas}

Dejours, C. (1993). Travail: usure mental. Paris: Bayard Edition. Vol 1.

Dejours, C. (1994). Psicodinâmica do trabalho: contribuiçoes da escola dejouriana à análise da relação prazer, sofrimento e trabalho. Betiol, 
M.I.S. (Coord.). São Paulo: Atlas.

Dejours, C. (1998). Souffrance en France: la banalisation de l' injustice sociale. Paris: Éditions du Sevil.

Deal, T. \& Kennedy, A. (1982). Corporate culture: the rites and rituals of corporate life. Massachusets: Addison-Wesley.

Freitas, M. E. (1991). Cultura organizacional: formação, tipologia e impacto. São Paulo: MacGraw-Hill

Freud, S. (1930\1974). O mal estar na civilização. In: Pequena coleção das obras de Freud. Rio de Janeiro: Imago. Livro 8.

Gondin, M. G. C. (1996). Valores organizacionais: hierarquia dos valores do $\mathrm{CNPq}$ segundo a percepção dos seus servidores. Dissertação de mestrado. Universidade de Brasília.

Hofstede, G.; Neuijen, B.; Ohayv, D. e Sanders, G. (1990). Measuring organizational cultures: a qualitative and quantitative study across twenty cases. Administrative Science Quarterly. 35, 286 -316.

Judge, T. \& Bretz, R. D. (1992). Effects of work on job choise decisions. Journal of Applied Psychology. 77, 261-271.

Meglino, M. B.; Ravlin, C.; Adkins, C. (1989). A work values approach to corporate cultures: a field test of the values congruence process and its relationship to individual outcomes. Journal of Applied Psychology. 74(3), 424-432.

Mendes, A. M. (1995). Os novos paradigmas de organização do trabalho: implicações para saúde mental do trabalhador. Revista Brasileira de Saúde Ocupacional. $85 \backslash 86$ (1), 72-80.

Mendes, A. M. \& Abrahão, J. I. (1996). A influência da organização do trabalho nas vivências de prazersofrimento do trabalhador: uma abordagem psicodinâmica. Revista Psicologia: Teoria e Pesquisa, 26(2), 179-184.

Mendes, A. M. (1996). Comportamento defensivo: uma estratégia para enfrentar o sofrimento no trabalho. Revista de Psicologia da UFC, 13\14(1\2),27-32.

Mendes, A. B. (1999). Valores e vivências de prazer-sofrimento no contexto organizacional. Tese de doutorado. Brasília. UnB.

Mendes, A. M.; Morrone F. C.; Watrin, B.; Vieira G.; Santos M. e Brauer S. (2000). Escala de indicadores de prazer e sofrimento no trabalho (EIPST): Construção e validação. (resumo). In Sociedade Brasileira de Psicologia (org), XXX Reunião Anual de Psicologia (p.223). Brasília: SBP.

Pascale, R. (1984). Fitting new employees into the company culture. Fortune. Maio 28.

Ravlin, E. C. \& Meglino, B. M. (1989). The transitivity of work values hierarchical preferences ordering of socially desirable stimuli. Organizational Behavior and Human Decision Processes, 44, 494-508.
Ros, M.; Schwartz, S. H. \& Surbiss (1999). Basic values, work values and the meaning of work. Applied Psychology: An International Review, 48 (1), 49-71.

Schein, E. H.(1990). Organizational culture. American Psychologist, Freb, 45(2).109-119.

Schwartz, S. H. (1999). A theory of cultural values and some implications for words. Applied Psychology: An International Review, 48(1), 23-47.

Schwartz, S H. \& Ros, M. (1995). Values in the west: a theorical and empirical challenge to the individualismcollectivism cultural dimension. World Psychology, 1, 91122.

Shoskley, E. \& Morley, D. (1989). Adhering to organizational culture. What is it mean? Why does it matter? Group \& Organizational Studies, 14 (4), 483-500.

Shrivastava, P. (1985). Integrating strategy formulation with organizational culture. Administrative Science Quarterly, 28 (3), 78-98.

Tamayo, A. (1996). Valores organizacionais. In: Tamayo, A., Borges-Andrade, J. e Codo, W. (Orgs.). Trabalho, organizações e cultura. ANPEPP. São Paulo: Cooperativa de autores associados. Vol. 1, n.11.

Tamayo-Robayo, M. (1997). Relação entre a sindrome do burnout e os valores organizacionais. Dissertação de mestrado. Universidade de Brasília.

Tamayo, A.; Mendes, A M.; Paz, M. G. T. (2000). Inventário de valores organizacionais. Revista Esudos em psicologia, 5(2), 289-317.

Trice, M. H. \& Beyer, M.J. (1993). The culture of work organizational. New Jersey: Prentice-Hall.

Wiener, Y. (1988). Forms of values systems: a focus on organizational effectiveness and cultural change and maintenance. Academy of Management Review, 13 (4), 534545.

Zilbovicis, M. (1988). Cultura Organizacional e mudança tecnológica: estudo de caso de uma montadora de automóveis no Brasil. ANAIS: Padrões Tecnológicos e Política de Gestão de Processo de Trabalho na Industria Brasileira. Departamento de Sociologia. São Paulo: USP.

Recebido em 24/03/2001

Revisado em 15/05/2001

Aceito em 21/06/2001

Sobre os autores:

Ana Magnólia Mendes é Doutora em Psicologia pela Universidade de Brasília, 1999. Professora do Departamento de Psicologia Social e do Trabalho da Universidade de Brasília. Pesquisadora do CNPq.

Álvaro Tamayo é Doutor em Psicologia pela Université Catholique de Louvain, 1970. Professor do Departamento de Psicologia Social e do Trabalho da Universidade de Brasília. Pesquisador do CNPq. 